\title{
Omega-3 supplementation on inflammatory markers in patients with chronic Chagas cardiomyopathy: a randomized clinical study
}

Paula Simplício da Silva ${ }^{1 *}$, Mauro Felippe Felix Mediano², Gilberto Marcelo Sperandio da Silva², Patricia Dias de Brito ${ }^{1}$, Claudia Santos de Aguiar Cardoso ${ }^{1}$, Cristiane Fonseca de Almeida', Luiz Henrique Conde Sangenis², Roberta Olmo Pinheiro ${ }^{3}$, Alejandro Marcel Hasslocher-Moreno ${ }^{2}$, Pedro Emmanuel Alvarenga Americano do Brasil ${ }^{2}$ and Andrea Silvestre de Sousa ${ }^{2}$

\begin{abstract}
Background: Several studies have been focusing on the effect of omega-3 polyunsaturated fatty acids on modulation of inflammatory markers in several cardiopathies. Although immunoregulatory dysfunction has been associated to the chronic cardiac involvement in Chagas disease, there is no study examining the effects of omega-3 supplementation in these patients. We investigated the effects of omega-3 PUFAs on markers of inflammation and lipid profile in chronic Chagas cardiomyopathy patients.
\end{abstract}

Methods: The present study was a single-center double-blind clinical trial including patients with chronic Chagas cardiomyopathy. Patients were randomly assigned to receive omega-3 PUFAs capsules (1.8g EPA and 1.2g DHA) or placebo (corn oil) during an 8-week period. Cytokines, fasting glucose, lipid, and anthropometric profiles were evaluated.

Results: Forty-two patients (23 women and 19 men) were included in the study and there were only two losses to follow-up during the 8-week period. Most of sociodemographic and clinical characteristics were similar between the groups at baseline, except for the cytokines IL-1 $\beta, I L-6, I L-8, I L-10, I L-17 a$, and IFNy. The omega-3 PUFAs group demonstrated greater improvements in serum triglycerides ( -21.1 vs. $-4.1 ; p=0.05)$ and IL-10 levels $(-10.6$ vs. $-35.7 ; p=0.01)$ in comparison to controls after 8 weeks of intervention. No further differences were observed between groups.

Conclusion: Omega-3 PUFAs supplementation may favorably affect lipid and inflammatory profile in chronic Chagas cardiomyopathy patients, demonstrated by a decrease in triglycerides and improvements on IL-10 concentration. Further studies examining the clinical effects of omega-3 fatty acids supplementation in chronic Chagas cardiomyopathy are necessary.

Trial registration: NCT01863576.

Keywords: Chagas disease, Chagas cardiomyopathy, Omega-3, Cytokines, Lipid profile

\footnotetext{
* Correspondence: paula.simplicio@ini.fiocruz.br

'Serviço de Nutrição, Instituto Nacional de Infectologia Evandro Chagas,

Fundação Oswaldo Cruz, Av. Brasil 4365, Manguinhos, Rio de Janeiro, Brazil

Full list of author information is available at the end of the article
} 


\section{Background}

Chagas disease is a neglected disease infection caused by the protozoan Trypanosoma cruzi [1], affecting approximately 6 million people worldwide, mostly in Latin America [2]. Over the last years, migratory movements have been increasing the number of cases reported in non-endemic countries such as North America and Europe [3], with the possibility of non-vectorial transmission in these countries.

Chronic Chagas cardiomyopathy (CCC) is an important clinical manifestation of Chagas disease, including heart failure, ventricular and atrial arrhythmias, atrioventricular blocks, thromboembolism, stroke and sudden death [4-6]. The degree of heart dysfunction is associated with a progressive inflammatory reaction, which is characterized by a myocarditis with multifocal mononuclear inflammatory infiltrates, a varied degree of myocardium fibrosis, a constant low-grade tissue parasitism, and a low or undetectable parasitemia [7-10].

Immunoregulatory dysfunction has been speculated as an important mechanism related to the progression of Chagas disease [11]. The unbalance between excessive proinflammatory cytokines and decreased anti-inflammatory cytokines produces a low-grade pro-inflammatory state that could be associated to the progression of disease and the increased morbidity and mortality rates among these patients $[11,12]$.

Several studies have been focusing on the effect of nutritional interventions on modulation of inflammatory markers, including omega-3 polyunsaturated fatty acids (PUFAs) [13-15]. A study including 133 patients with a diagnosis of non-ischemic dilated cardiomyopathy and left ventricular systolic dysfunction (defined as an ejection fraction $\leq 45 \%$ ) showed that omega-3 PUFAs supplementation promoted improvements on inflammatory markers, cardiac function and functional capacity in comparison to placebo after 12 months of follow-up. In addition, the hospitalization rates were $6 \%$ in the omega-3 PUFAs and $30 \%$ in the placebo group, highlighting the important clinical benefits promoted by omega-3 PUFAs supplementation among patients with systolic dysfunction [14].

The anti-inflammatory properties of omega-3 PUFAs are associated with short and long-term cardioprotective effects $[16,17]$. The GISSI-HF trial, the major study evaluating the long-term effects of omega-3 PUFAs in individuals with chronic heart failure ( $\mathrm{CHF})$, showed a significant decrease in all-cause mortality and a composite of cardiovascular mortality and cardiovascular hospitalization in omega-3 PUFAs group in comparison to controls [13]. In addition, another study including 250 CHF patients achieved important improvements on cardiac function among those who received omega-3 PUFAs supplementation after six months of follow-up [18].
In this context, although several studies have been showing beneficial effects of omega-3 PUFAs on inflammatory and biochemical markers in individuals with $\mathrm{CHF}$, none of them included patients with CCC and the potential effects of omega-3 PUFAs in this population is still unknown. In this study, we sought to investigate the effects of omega-3 PUFAs on inflammatory markers and lipid profile in CCC patients.

\section{Methods \\ Study design}

The present study was a single-center double-blind clinical trial conducted at the Evandro Chagas National Institute of Infectious Disease (Rio de Janeiro, Brazil). The full description of the study protocol have been published previously [19].

Patients aging $>18$ years, with a diagnosis of $\mathrm{CCC}$ on stages B, C or D according to the current Brazilian Chagas disease consensus were included in the study [20]. The exclusion criteria were diarrheal disease, inflammatory bowel syndrome, the use of anti-inflammatory drugs, pregnant and lactating women, vitamin mineral or omega-3 PUFAs supplementation during the previous 30 days before the beginning of study, and the presence of cardiomyopathies other than CCC. All patients received information about the goals and the procedures of the study and signed an informed consent. The research protocol was approved by the local Institutional Review Board (CAAE-0037.0.009.000-10) and was registered in the ClinicalTrials.gov website (NCT01863576).

\section{Intervention}

Patients were randomly assigned in a blinded fashion in a 1:1 ratio using sequentially numbered opaque sealed envelopes into one of the two groups: 1) an intervention group that received omega-3 PUFAs capsules (1.8g eicosapentaenoic acid and 1.2gdocosahexaenoic acid); 2) control group that received placebo (corn oil). The randomization list was computer-generated with blocking in advance ( 4 or 6 patients). The single researcher responsible for placing participants on study had no participation in the process of randomization.

Participants were counseled to take five omega-3 or placebo capsules per day (three in the morning and two the evening) and were followed during an 8-week period. They were also advised to not change their usual dietary habits during the study. The capsules were dispensed at the beginning of the study and after 4 weeks. The returned capsules were checked at weeks 4 and 8 to evaluate compliance to treatment.

The blister packaging and gel capsules of omega-3 PUFAs and placebo were identical and identified by the lot number on the package (Relthy Laboratories Ltda; Brazilian Health Surveillance Agency registration: 6.2582.0022.001-1). 


\section{Measurements}

Sociodemographic (age, sex, race and schooling) and clinical characteristics (NYHA functional class, alcohol consumption, smoking, and drugs prescription) were assessed at baseline using the information contained in the medical records. Food consumption and anthropometric measurements were evaluated thrice (baseline, after 4 and 8 weeks) whereas biochemical markers and cytokines were evaluated twice during the study (baseline and after 8 weeks).

Food intake was estimated by a single-trained nutritionist using the mean of 24-h dietary recalls assessed in three different moments during the study (weeks 0,4 , and 8) [21]. The 24-h dietary recall is a structured interview intended to capture detailed information about all foods and beverages consumed by the respondent in the past 24 $\mathrm{h}$ from midnight to midnight of the previous day. The dietitians entered dietary data directly into a nutrition analysis software (Diet Win plus $3.0^{\circ}$ software package, Brubins Ltda.) to obtain the amount of carbohydrates, proteins, lipids, fiber, cholesterol, fatty acids (monounsaturated, polyunsaturated, saturated) and total calories intake.

The anthropometric evaluation consisted of measurements of height, body weight, waist and hip circumferences, tricipital skinfold thickness and arm circumference. Height and body weight were assessed with minimal clothes and without shoes using a calibrated digital scale coupled with a stadiometer. The body mass index (BMI) was calculated to determine the nutritional status [22]. Waist and hip circumferences were taken at the smallest girth of the waist and largest girth of the hip, respectively. An inelastic measuring tape was used to measure the mid-upper arm circumference and a scientific calibrated caliper $\left(\right.$ Cescorf $\left.^{\circledR}\right)$ was used to measure triceps skinfold thickness. The results were compared to the standards values established according to age $[23,24]$.

Blood samples were collected after $12 \mathrm{~h}$ of overnight fasting. Aliquots of plasma and serum were isolated from the blood samples and frozen at $-70 \mathrm{C}$ within $2 \mathrm{~h}$ of being drawn. Total cholesterol, triglycerides, HDL-cholesterol, and glucose were measured using Siemens Dimension ${ }^{\bullet}$ reagent cartridge with an intra and inter-assay coefficients of variability $<5 \%$. The LDL- and VLDL-cholesterol concentrations were calculated according to the Friedewald equation based on the triglycerides measures [25]. Cytokine serum levels (IL-1 $\beta$, IL-4, IL-6, IL-8, IL-10, IL-17- $\alpha$, IL-33 TNF- $\alpha$ and IFN- $\gamma$ ) were measured in the serum of patients using specific sandwich enzyme-linked immunosorbent assays. Capture and detection antibodies were obtained from eBioscience kits (San Diego, CA, USA). The tests were conducted according to the manufacturer's instructions.

\section{Blinding}

All sociodemographic, clinical characteristics, food consumption and anthropometric measurements, biochemical markers and cytokines measurements were performed in a blinded fashion by the same single evaluator.

\section{Sample size}

Considering a prevalence of $60 \%$ of inadequate omega-3 PUFAs intake [26], IFNy values of 3986 (738) $\mathrm{pg} / \mathrm{mL}$ at baseline and IFN $\gamma$ values of 2922 (1275) pg/mL after

Table 1 Baseline characteristics of patients included in the study $(n=42)$

\begin{tabular}{|c|c|c|}
\hline Variable & Control $(n=21)$ & Intervention $(n=21)$ \\
\hline Age (years) & $55.0(9.5)$ & $58.6(11.0)$ \\
\hline \multicolumn{3}{|l|}{ Sex } \\
\hline Male & $38.1 \%(n=08)$ & $52.4 \%(n=11)$ \\
\hline Female & $61.9 \%(n=13)$ & $47.6 \%(n=10)$ \\
\hline Income (Reais, R\$) & $1028.2(556.9)$ & $1168.9(602.8)$ \\
\hline \multicolumn{3}{|l|}{ Schooling } \\
\hline Illiterate & $14.3 \%(n=3)$ & $19.0 \%(n=4)$ \\
\hline Incomplete elementary school & $61.9 \%(n=13)$ & $61.9 \%(n=13)$ \\
\hline Complete elementary school & $14.3 \%(n=03)$ & $4.8 \%(n=01)$ \\
\hline Incomplete high school & $4.8 \%(n=01)$ & $9.5 \%(n=02)$ \\
\hline High school & $4.8 \%(n=01)$ & $4.8 \%(n=01)$ \\
\hline Ejection fraction (\%) & $45.7(14.8)$ & $44.2(14.0)$ \\
\hline Weight (kg) & $67.3(11.3)$ & $69.1(14.1)$ \\
\hline Height (m) & $1.56(0.1)$ & $1.60(0.1)$ \\
\hline Body mass index $\left(\mathrm{kg} / \mathrm{m}^{2}\right)$ & $27.7(5.2)$ & $27.0(4.7)$ \\
\hline Brachial circumference $(\mathrm{cm})$ & $29.4(4.2)$ & $28.3(3.9)$ \\
\hline Triceps skinfold thickness (mm) & $18.4(11.1)$ & $15.1(7.7)$ \\
\hline $\begin{array}{l}\text { Brachial muscle circumference } \\
(\mathrm{cm})\end{array}$ & $23.3(2.6)$ & $23.7(2.8)$ \\
\hline Waist circumference (cm) & $87.0(9.6)$ & $89.0(12.8)$ \\
\hline Hip circumference (cm) & $100.4(10.0)$ & $99.5(10.9)$ \\
\hline Triacylglycerol (mg/dL) & $93.8(39.5)$ & $97.7(50.9)$ \\
\hline Total cholesterol (mg/dL) & $166.7(27.5)$ & $171.7(38.8)$ \\
\hline HDL-cholesterol (mg/dL) & $50.4(13.4)$ & $51.1(16.2)$ \\
\hline LDL-cholesterol (mg/dL) & $97.5(23.2)$ & $105.8(32.9)$ \\
\hline VLDL-cholesterol (mg/dL) & $18.8(8.0)$ & $18.8(9.1)$ \\
\hline Glucose (mg/dL) & $93.8(9.5)$ & $95.6(8.0)$ \\
\hline Tumour necrosis factor- $a$ & $66.4(20.9)$ & $65.6(19.1)$ \\
\hline Interleukin-4 & $22.2(6.6)$ & $23.1(9.2)$ \\
\hline Interleukin-6 & $335.4(486.9)$ & $163.9(142.1)$ \\
\hline Interleukin-8 & $84.8(58.1)$ & $59.8(26.2)$ \\
\hline Interleukin-10 & $105.2(71.2)$ & $70.4(28.4)$ \\
\hline Interleukin-33 & $77.2(23.6)$ & $86.7(66.5)$ \\
\hline Interleukin-1b & $21.1(8.9)$ & $18.9(4.4)$ \\
\hline Interleukin-17a & $5082.3(5086.2)$ & $3940.4(4685.9)$ \\
\hline Interferon- $\gamma$ & $75.5(112.9)$ & $47.8(84.2)$ \\
\hline
\end{tabular}

Data are expressed as means (standard deviation) and prevalence of frequency 
omega-3 PUFA supplementation [27], and assuming 80\% of power and $5 \%$ of significance level, the minimum estimated sample size was 40 patients (20 in each group).

\section{Data analysis}

Baseline characteristics of the two groups were expressed as the mean (standard deviation) for continuous and percentages for categorical variables. Skewness and Kurtosis test was performed to evaluate the normality of data that were log-transformed in case of skewed distribution. Longitudinal changes between groups for all variables were examined using linear mixed models, which considers the treatment assignment and includes all observations of each participant regardless of loss to follow-up or compliance, characterizing an intention-totreat analysis. The model included intervention group, time and time $\mathrm{X}$ intervention group interaction. The term of interest was time $\mathrm{X}$ group interaction, which estimates the rate of changes in the outcomes. The longitudinal model was adjusted for baseline values of the dependent variable in case of major unbalance for baseline values. Residual plots of all models were examined and did not show major deviations from regression assumptions. All statistical analysis were accomplished using Stata 13.0 software (College Station, TX, 2013) and significance level was set at $p \leq 0.05$ for all analyses.

\section{Results}

A total of 42 patients (23 women and 19 men) were included in the study from May, 2013 to September, 2013. Baseline characteristics of patients randomized to the two groups are shown in Table 1. Most of sociodemographic and clinical characteristics were similar between

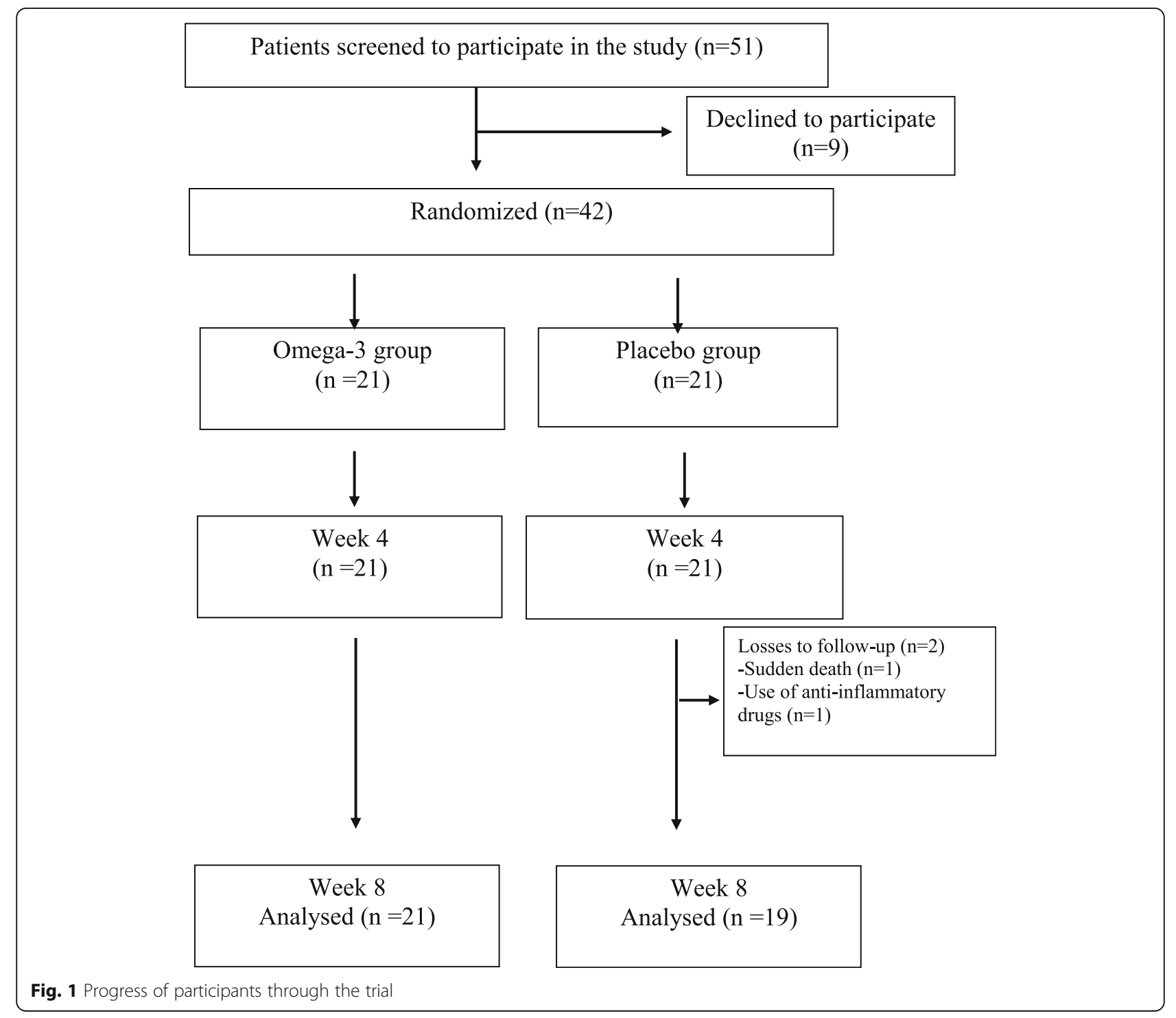


the groups at baseline, except for the cytokines IL-1 $\beta$, IL-6, IL-8, IL-10, IL-17 $\alpha$, and IFN $\gamma$.

The majority of patients were in use of beta-blockers $(69.1 \%)$ and $85 \%$ were in use of angiotensin-converting enzyme inhibitors/angiotensin II receptor blockers with no major treatment differences between groups $(81 \%, n$ $=17$ for control and 90.5\%, $n=19$ for intervention), except the omega-3 PUFAs intervention.

Losses to follow-up during the 8-week period were 5\% (one sudden death during a recreational soccer game and one patient in use of anti-inflammatory drug during the study period, both not related to the omega-3 PUFAs intervention) and the flow chart of patients included in the study is depicted in Fig. 1. Compliance levels were high for both groups, with no statistical differences to the percentage of ingested capsules at the end of followup $(92.6 \%$ for placebo vs. $94.4 \%$ for omega-3 PUFAs; $p=0.41$ ).

Crude means and changes from baseline for anthropometric, biochemical, and inflammatory variables are depicted in Tables 2, 3 and 4. No significant differences were found for carbohydrates, proteins, lipids, fiber, cholesterol, fatty acids (monounsaturated, polyunsaturated, saturated) and total calories intake between placebo and omega-3 PUFA groups during the entire follow-up period (Table 5). There were no differences between groups for any of the investigated anthropometric variables after 4 and 8 weeks of follow-up. However, the omega-3 PUFAs group demonstrated improvements in serum triglycerides $(-21.1$ vs. $-4.1 ; p=0.05)$ and IL10 levels $(-10.6$ vs. $-35.7 ; p=0.01)$ in comparison to controls at the end of study ( 8 weeks). No further differences were observed between groups for biochemical and inflammatory markers. No adverse reactions associated with omega-3 PUFAs supplementation were reported during the follow-up.

\section{Discussion}

Omega-3 PUFAs supplementation has been demonstrating significant effects on prevention and treatment of many chronic health conditions, including heart failure $[13,28,29]$. A prospective cohort study conducted by Mozzafarian et al.[30] demonstrated an important association between an increased omega-3 PUFAs concentration

Table 2 Crude means (standard deviation) and estimated changes from baseline $(\Delta)$ for anthropometric variables during the follow-up

\begin{tabular}{|c|c|c|c|c|c|c|}
\hline & \multicolumn{3}{|l|}{4 week } & \multicolumn{3}{|l|}{8 week } \\
\hline & \multicolumn{3}{|c|}{ (Control = 20; Intervention = 21) } & \multicolumn{3}{|c|}{$($ Control = 20; Intervention = 21) } \\
\hline & Mean (DP) & $\Delta^{\mathrm{a}}$ & $p^{a}$ & Mean (DP) & $\Delta^{\mathrm{a}}$ & $p^{a}$ \\
\hline \multicolumn{7}{|l|}{ Weight (kg) } \\
\hline Control & $67.9(11.6)$ & +0.43 & & $68.7(10.2)$ & +0.52 & \\
\hline Intervention & $68.2(13.7)$ & +0.46 & 0.946 & $69.9(13.4)$ & +0.84 & 0.386 \\
\hline \multicolumn{7}{|c|}{ Body mass index $\left(\mathrm{kg} / \mathrm{m}^{2}\right)$} \\
\hline Control & $28.1(5.2)$ & +0.18 & & $28.4(4.7)$ & +0.36 & \\
\hline Intervention & $26.7(4.7)$ & 0.00 & 0.438 & $27.2(4.5)$ & +0.21 & 0.542 \\
\hline \multicolumn{7}{|c|}{ Arm circumference $(\mathrm{cm})$} \\
\hline Control & $29.6(5.0)$ & +0.04 & & $29.9(4.6)$ & +0.16 & \\
\hline Intervention & $28.1(4.2)$ & +0.03 & 0.986 & $28.6(3.7)$ & +0.33 & 0.703 \\
\hline \multicolumn{7}{|c|}{ Tricipital skinfold thickness (mm) } \\
\hline Control & $18.4(9.9)$ & -0.29 & & $18.5(10.1)$ & -0.17 & \\
\hline Intervention & $15.7(8.2)$ & +0.26 & 0.057 & $15.1(7.6)$ & +0.14 & 0.261 \\
\hline \multicolumn{7}{|c|}{ Arm circumference $(\mathrm{cm})$} \\
\hline Control & $23.3(2.3)$ & 0.00 & & $24.0(2.8)$ & +0.48 & \\
\hline Intervention & $23.6(2.9)$ & +0.09 & 0.840 & $23.8(2.7)$ & +0.08 & 0.323 \\
\hline \multicolumn{7}{|c|}{ Waist circumference (cm) } \\
\hline Control & $87.1(9.5)$ & -0.28 & & $88.1(8.5)$ & +0.59 & \\
\hline Intervention & $88.2(12.7)$ & +0.20 & 0.263 & $89.1(12.6)$ & +0.12 & 0.259 \\
\hline \multicolumn{7}{|c|}{ Hip circumference $(\mathrm{cm})$} \\
\hline Control & $100.3(10.1)$ & -0.25 & & $101.3(9.1)$ & +0.11 & \\
\hline Intervention & $99.0(10.6)$ & +0.29 & 0.213 & $100.1(10.1)$ & +0.59 & 0.258 \\
\hline
\end{tabular}

aLinear mixed models include time, group and time $\mathrm{x}$ group interaction adjusted for baseline values 
Table 3 Crude means (standard deviation) and estimated changes from baseline $(\Delta)$ for biochemical variables

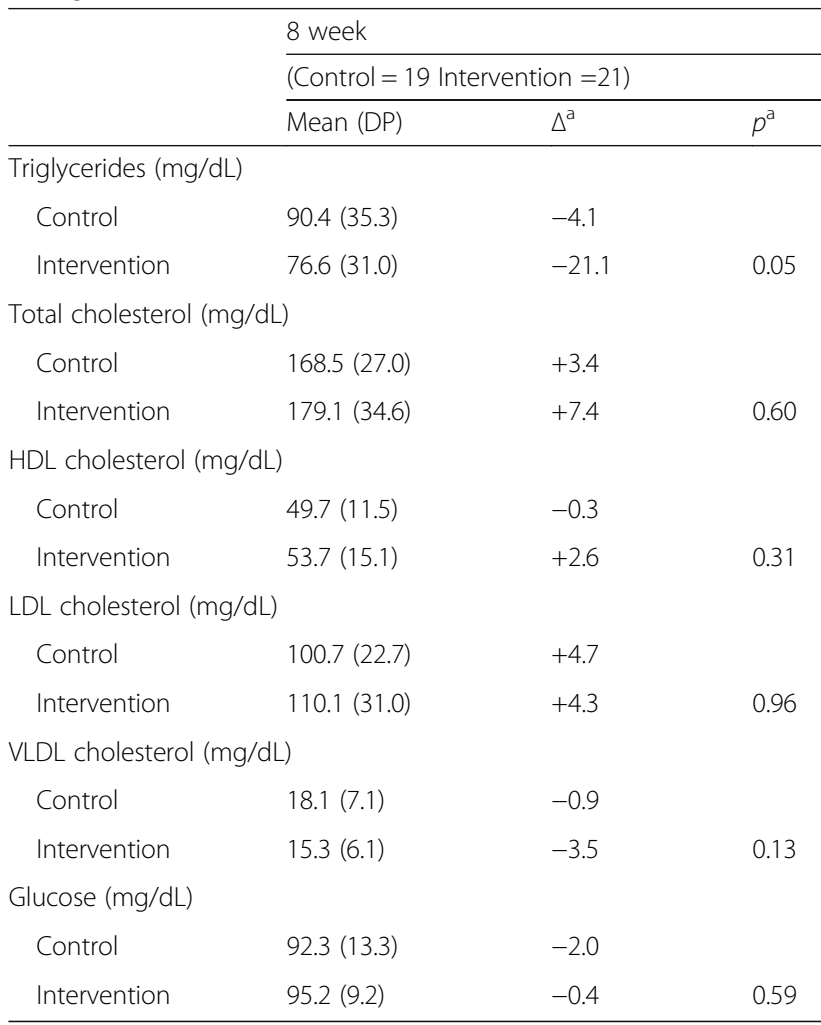

ainear mixed models include time, treatment and time $\mathrm{x}$ treatment interaction, adjusted for baseline values

and a lower incidence of congestive heart failure (HR 0.70; CI 95\% 0.49-0.99) following 2735 U.S. adults free of heart disease during 14 years.

The physiological effects of omega-3 PUFAs supplementation are associated to the incorporation of long chain omega-3 PUFAs into the cell membranes, modulating their biophysical properties and functionality. PUFAs are biosynthesis precursors of several important metabolites, especially eicosanoids (prostaglandins, leukotrienes, thromboxanes, lipoxins, and others), synthesized from arachidonic acid [31]. Omega-3 PUFAs reduce arachidonic acid content in cell membranes, resulting in the synthesis of eicosanoids that have greater antiinflammatory properties than the eicosanoids derived from omega-6 PUFAs [32]. The American College of Cardiology and American Heart Association (ACC/AHA) considers that omega-3 PUFAs supplementation is an important adjuvant in the heart failure therapy [33].

To our knowledge, the present study was the first one to demonstrate a reduction of serum triglycerides as result of the omega-3 PUFAs supplementation in patients with CCC. This is important given that elevated triglyceride levels are considered independent predictors of cardiovascular risk [34]. Results from preclinical and clinical studies suggested that omega-3 PUFAs decrease
Table 4 Crude means (standard deviation) and estimated changes from baseline $(\Delta)$ for inflammatory markers

\begin{tabular}{|c|c|c|c|}
\hline & 8 week & & \\
\hline & (Control = $19 \mathrm{In}$ & $n=21)$ & \\
\hline & Mean (DP) & $\Delta^{a}$ & $p^{a}$ \\
\hline Tumor necrosis & $(\mathrm{pg} / \mathrm{dL})$ & & \\
\hline Control & $84.5(51.2)$ & +19.4 & \\
\hline Intervention & $90.7(48.8)$ & +27.2 & 0.58 \\
\hline Interleukin-4 (pg & & & \\
\hline Control & $21.5(9.7)$ & -0.8 & \\
\hline Intervention & $20.7(6.7)$ & -1.5 & 0.67 \\
\hline Interleukin-6 (pg & & & \\
\hline Control & $67.3(80.2)$ & -228.1 & \\
\hline Intervention & $61.5(54.3)$ & -85.3 & 0.45 \\
\hline Interleukin-8 (pg & & & \\
\hline Control & $64.7(48.4)$ & -11.3 & \\
\hline Intervention & $53.6(12.4)$ & -3.3 & 0.47 \\
\hline Interleukin-10 (p & & & \\
\hline Control & $53.8(7.1)$ & -35.7 & \\
\hline Intervention & $56.3(8.3)$ & -10.6 & 0.01 \\
\hline Interleukin-33 (p & & & \\
\hline Control & 70.4 (15.6) & -5.2 & \\
\hline Intervention & $69.4(17.0)$ & -5.5 & 0.97 \\
\hline Interleukin-1b (p & & & \\
\hline Control & $18.0(3.4)$ & -2.3 & \\
\hline Intervention & $17.9(3.4)$ & -0.6 & 0.24 \\
\hline Interleukin-17a ( & & & \\
\hline Control & $3649.2(4254.2)$ & +951.5 & \\
\hline Intervention & $6130.0(7644.2)$ & +3621.7 & 0.24 \\
\hline Interferon- $\gamma$ (pg/ & & & \\
\hline Control & $82.6(112.3)$ & +9.3 & \\
\hline Intervention & $53.2(100.7)$ & +7.1 & 0.90 \\
\hline
\end{tabular}

ainear mixed models include time, treatment and time $\mathrm{x}$ treatment interaction, adjusted for baseline values

serum triglycerides concentrations by reducing its synthesis, reducing the triglyceride incorporation into VLDL, reducing triglyceride secretion, and enhancing triglyceride clearance from VLDL particles [15]. In a study conducted by Kastelein et al. [35], 399 subjects with severe hypertriglyceridemia $(\geq 500 \mathrm{mg} / \mathrm{dL}$ to $<2000 \mathrm{mg} / \mathrm{dL}$ ) were randomly assigned to receive placebo (olive oil $4 \mathrm{~g} / \mathrm{d}$ ) or omega-3 PUFAs supplementation at different dosages (2 $\mathrm{g} / \mathrm{d}, 3 \mathrm{~g} / \mathrm{d}$ or $4 \mathrm{~g} / \mathrm{d}$ ). Fasting serum triglycerides changed from baseline by $-25.9 \%$ ( $p<0.01$ vs. placebo), $-25.5 \%$ ( $p$ $<0.01$ vs. placebo), and $-30.9 \%$ ( $p<0.001$ vs. placebo) for $2 \mathrm{~g} / \mathrm{d}, 3 \mathrm{~g} / \mathrm{d}$, and $4 \mathrm{~g} / \mathrm{d}$ of omega-3 PUFAs, respectively. In another study, patients with metabolic syndrome treated with $2 \mathrm{~g} / \mathrm{d}$ of omega-3 PUFAs also found a decrease in triglycerides levels from day 0 to 28 and $84(p<0.01)$ and in 
Table 5 Means (standard deviation) of dietary variables and energy intake $(n=42)$

\begin{tabular}{llll}
\hline Variable & Control $(n=21)$ & Intervention $(n=21)$ & $p$ \\
\hline Energy (kcal) & $1487.59(407.36)$ & $1480.18(419.08)$ & 0.95 \\
Carbohydrates (g) & $202.75(49.04)$ & $200.59(49.71)$ & 0.89 \\
Protein (g) & $72.30(27.77)$ & $70.99(26.00)$ & 0.88 \\
Total fat (g) & $42.94(19.93)$ & $43.03(19.50)$ & 0.99 \\
Fiber (g) & $18.48(7.01)$ & $19.22(7.75)$ & 0.75 \\
Cholesterol (mg) & $215.60(129.55)$ & $205.70(87.81)$ & 0.77 \\
Saturated fatty acids (g) & $12.60(5.93)$ & $13.81(7.62)$ & 0.57 \\
Monounsaturated fatty & $12.36(7.82)$ & $13.15(8.02)$ & 0.75 \\
acids (g) & & & \\
Polyunsaturated fatty & $9.54(5.70)$ & $8.41(3.80)$ & 0.45 \\
acids (g) & & & \\
Trans fatty acids (g) & $0.65(0.58)$ & $0.80(0.76)$ & 0.48 \\
Linoleic acid, n-6 (g) & $6.17(4.24)$ & $5.65(2.99)$ & 0.65 \\
Alfa-linoleic acid, $\mathrm{n}-3$ (g) & $0.72(0.80)$ & $0.56(0.32)$ & 0.42 \\
\hline
\end{tabular}

serum total cholesterol levels $(p<0.01)$, highlighting the importance of omega-3 supplementation on improvements of lipid profile [36].

Most of studies involving omega-3 supplementation have been demonstrating a decreased production of proinflammatory cytokines such as TNF- $\alpha$, IL- $1 \beta$ and IL-6 $[37,38]$. Similar results were observed in a meta-analysis that evaluated of the effects of fish oil supplementation in patients with chronic heart failure, showing that circulating TNF $\alpha$, IL-1 $\beta$, and IL- 6 decreased after 3 to 12 months of follow-up [39].

In our study, omega-3 PUFAs were able to attenuate the decrease observed in the IL-10 concentration (an anti-inflammatory cytokine) at the end of follow-up, showing an important positive effect of omega-3 PUFAs supplementation on inflammatory modulation in patients with CCC. Although these results have been demonstrated by studies conducted in several different populations [40-42], those examining the effects of omega-3 PUFAs supplementation on IL-10 in patients with HF presented controversial results. For instance, no changes for IL-10 were observed in a study including 16 male patients with HF that received omega-3 PUFAs supplementation during 60 days [43]. In addition, another study including 190 patients with paroxysmal or persistent atrial fibrillation showed no significant effects of omega-3 PUFAs supplementation on IL-10 concentration after a 6-month period [44].

Specific immune expression of anti-inflammatory and pro-inflammatory cytokines may play a relevant role in the clinical presentation of chronic Chagas disease [45]. $T$. cruzi infection induces a strong inflammatory response dominated by the Th1 pattern, with the IFN- $\gamma$ and TNF- $\alpha$ expressions regulated by the IL-10 production [46]. The immunological balance in patients with indeterminate form has a high expression of IL-10, whereas cardiac form is associated with a reduction of IL-10 production and increased production of TNF- $\alpha$ and IFN- $\gamma$. The proinflammatory cytokines IL-12, IFN- $\gamma$ and TNF- $\alpha$ activate macrophages to promote parasite killing through the production of trypanocidal radicals. These cytokines also acts as a positive feedback for Th1 differentiation. Th1 cells orchestrate an expressive CD8+ T cell response, causing tissue damage and fibrosis $[46,47]$. Correlation analysis showed that higher IL-10 expression was associated with better cardiac function, as determined by left ventricular ejection fraction and left ventricular diastolic diameter values [12]. In this way, the results of our study demonstrate a positive influence of omega-3 PUFAs supplementation upon inflammatory profile in patients with CCC.

The present study has some limitations. Although sample size has been calculated to identify longitudinal statistical differences for cytokines between omega-3 PUFAs and placebo groups, this small number of patients may have contributed to the differences observed between groups for some cytokines at baseline. However, we included baseline values in longitudinal models in order to minimize the influence of baseline imbalance on statistical analysis. The short-term follow-up period did not allow us to evaluate clinical outcomes, though that was not the scope of the present study. In addition, the lack of a health control group and the absence of a physical activity measurement could make the interpretation of results difficult. Strengths included the very low frequency of losses to follow-up and the high compliance rates, which are very difficult to achieve in clinical trials [48].

To conclude, omega-3 PUFAs supplementation may favorably affect lipid and inflammatory profile in chronic Chagas cardiomyopathy patients, demonstrated by the decrease in triglycerides and improvements on IL-10 concentration. This results highlights to the importance of omega-3 PUFAs supplementation as a new coadjuvant strategy to treat patients with CCC, promoting a better control of inflammatory parameters and triglycerides. Studies evaluating the effects of increased consumption of omega-3 rich foods as well as the long-term effects of omega-3 PUFAs on inflammatory profile and clinical outcomes in CCC requires further investigation.

\section{Abbreviations}

BMI: Body mass index; CCC: Chronic Chagas cardiomyopathy; CHF: Chronic heart failure; PUFAs: Polyunsaturated fatty acids

\section{Acknowledgements}

The authors thanks to Evandro Chagas National Institute of Infectious Diseases and to Oswaldo Cruz Institute for clinical and laboratorial support. The authors also thanks to Relthy Laboratories Ltda for donations of placebo and omega-3 PUFAs capsules. Beneficiary of financial assistance from CAPES - Brazil. 


\section{Funding}

Evandro Chagas National Institute of Infectious Disease funded this study.

\section{Availability of data and materials}

The datasets used and/or analyzed during the current study will be available on clinicaltrials.gov according to their guidelines.

\section{Authors' contributions}

PSS was involved in trial design, project development, manuscript drafting, patient recruitment, and collected clinical data from the patients. ASS was involved in trial design, manuscript drafting and review, and collected clinical data from the patients. MFFM, GMSS, ROP, LHCS, AMHM and PEAAB were involved in manuscript drafting or review and collected clinical data from the patients. CSAC, CAF and PDB were involved in patient evaluations and collected clinical data from the patients. All authors read and approved the final version of manuscript.

\section{Competing interests}

The authors declare that they have no competing interests.

\section{Consent for publication}

Not applicable.

\section{Ethics approval and consent to participate}

The research protocol was approved by the local Institutional Review Board (CAAE-0037.0.009.000-10) and was registered in the ClinicalTrials.gov website (NCT01863576). All patients received information about the goals and the procedures of the study and signed an informed consent.

\section{Publisher's Note}

Springer Nature remains neutral with regard to jurisdictional claims in published maps and institutional affiliations.

\section{Author details}

${ }^{1}$ Serviço de Nutrição, Instituto Nacional de Infectologia Evandro Chagas, Fundação Oswaldo Cruz, Av. Brasil 4365, Manguinhos, Rio de Janeiro, Brazil. ${ }^{2}$ Laboratório de Pesquisa Clínica em Doença de Chagas, Instituto de Pesquisa Clínica Evandro Chagas, Fundação Oswaldo Cruz, Av. Brasil 4365, Manguinhos, Rio de Janeiro, Brazil. ${ }^{3}$ Laboratório de Hanseníase, Instituto Oswaldo Cruz, Fundação Oswaldo Cruz, Av. Brasil 4365, Manguinhos, Rio de Janeiro, Brazil.

Received: 10 March 2017 Accepted: 5 June 2017 Published online: 09 June 2017

\section{References}

1. Rassi Jr A, Rassi A, Marcondes de Rezende J. American Trypanosomiasis (Chagas Disease). Infect Dis Clin North Am. 2012;26:275-91.

2. $\mathrm{WHO} \mid$ Chagas disease (American trypanosomiasis) [Internet]. WHO. [cited 2017 Feb 19]. Available from: http://www.who.int/mediacentre/factsheets/ fs340/en/

3. Coura JR, Viñas PA. Chagas disease: a new worldwide challenge. Nature. 2010;465:S6-7.

4. Marin Neto JA, Simões MV, Sarabanda AV. Chagas' heart disease. Arq Bras Cardiol. 1999;72:247-80.

5. Rocha MOC, Ribeiro ALP, Teixeira MM. Clinical management of chronic Chagas cardiomyopathy. Front Biosci J Virtual Libr. 2003;8:e44-54.

6. Rassi A, Rassi A, Marin-Neto JA. Chagas disease. Lancet. 2010:375:1388-402.

7. Punukollu G, Gowda RM, Khan IA, Navarro VS, Vasavada BC. Clinical aspects of the Chagas' heart disease. Int J Cardiol. 2007:115:279-83.

8. Ribeiro AL, Nunes MP, Teixeira MM, Rocha MOC. Diagnosis and management of Chagas disease and cardiomyopathy. Nat Rev Cardiol. 2012;9:576-89.

9. Cunha-Neto E, Chevillard C. Chagas Disease Cardiomyopathy: Immunopathology and Genetics. Mediators Inflamm. 2014;2014:e683230.

10. Pereira $I R$, Vilar-Pereira $G$, da Silva AA, Lannes-Vieira J. Severity of chronic experimental Chagas' heart disease parallels tumour necrosis factor and nitric oxide levels in the serum: models of mild and severe disease. Mem Inst Oswaldo Cruz. 2014;109:289-98.

11. Chaves AT, de Assis Silva Gomes Estanislau J, Fiuza JA, Carvalho AT, Ferreira KS, Fares RCG, et al. Immunoregulatory mechanisms in Chagas disease: modulation of apoptosis in T-cell mediated immune responses. BMC Infect Dis. 2016;16:191.

12. Sousa GR, Gomes JAS, Fares RCG, de Damásio MP S, Chaves AT, Ferreira KS, et al. Plasma Cytokine Expression Is Associated with Cardiac Morbidity in Chagas Disease. PLoS One. 2014;9:e87082.

13. Tavazzi L, Maggioni AP, Marchioli R, Barlera S, Franzosi MG, Latini R, et al. Effect of $n-3$ polyunsaturated fatty acids in patients with chronic heart failure (the GISSI-HF trial): a randomised, double-blind, placebo-controlled trial. Lancet. 2008;372:1223-30.

14. Nodari S, Triggiani M, Campia U, Manerba A, Milesi G, Cesana BM, et al. Effects of n-3 polyunsaturated fatty acids on left ventricular function and functional capacity in patients with dilated cardiomyopathy. J Am Coll Cardiol. 2011:57:870-9.

15. Backes J, Anzalone D, Hilleman D, Catini J. The clinical relevance of omega-3 fatty acids in the management of hypertriglyceridemia. Lipids Health Dis. 2016;15:118

16. Blondeau $\mathrm{N}$. The nutraceutical potential of omega-3 alpha-linolenic acid in reducing the consequences of stroke. Biochimie. 2016;120:49-55.

17. Endo J, Arita M. Cardioprotective mechanism of omega-3 polyunsaturated fatty acids. J Cardiol. 2016;67:22-7.

18. Chrysohoou C, Metallinos G, Georgiopoulos G, Mendrinos D, Papanikolaou A, Magkas N, et al. Short term omega-3 polyunsaturated fatty acid supplementation induces favorable changes in right ventricle function and diastolic filling pressure in patients with chronic heart failure; A randomized clinical trial. Vascul Pharmacol. 2016;79:43-50.

19. Silva PS, da Silva GM S, de Souza AP, Cardoso CSA, Fonseca CA, Brito PD, et al. Effects of omega-3 polyunsaturated fatty acid supplementation in patients with chronic chagasic cardiomyopathy: study protocol for a randomized controlled trial. Trials. 2013;14:379.

20. Dias JCP, Ramos AN, Gontijo ED, Luquetti A, Shikanai-Yasuda MA, Coura JR, et al. 2 nd Brazilian Consensus on Chagas Disease, 2015. Rev Soc Bras Med Trop. 2016:49Suppl 1:3-60.

21. Slater B, Marchioni DL, Fisberg RM. Estimating prevalence of inadequate nutrient intake. Rev Saude Publica. 2004:38:599-605.

22. Obesity: preventing and managing the global epidemic. Report of a WHO consultation. World Health Organ Tech Rep Ser. 2000;894:i-xii, 1-253.

23. Frisancho AR. New norms of upper limb fat and muscle areas for assessment of nutritional status. Am J Clin Nutr. 1981:34:2540-5.

24. Frisancho AR. Anthropometric standards for the assessment of growth and nutritional status. University of Michigan. 1990.

25. Friedewald WT, Levy RI, Fredrickson DS. Estimation of the concentration of low-density lipoprotein cholesterol in plasma, without use of the preparative ultracentrifuge. Clin Chem. 1972;18:499-502.

26. Salvador MB, Sarkis KS, Silva RG, de Zerbini CA F, Martini LA. Avaliação da ingestão de ácidos graxos, antioxidantes e da composição corporal em mulheres com artrite reumatóide; Fatty acids, antioxidants and body composition evaluation in woman with reumathoid arthritis. Nutr Rev Soc Bras Aliment Nutr. 2008;33:17-30

27. Kew S, Mesa MD, Tricon S, Buckley R, Minihane AM, Yaqoob P. Effects of oils rich in eicosapentaenoic and docosahexaenoic acids on immune cell composition and function in healthy humans. Am J Clin Nutr. 2004:79:674-81.

28. Mozaffarian D, Bryson CL, Lemaitre RN, Burke GL, Siscovick DS. Fish Intake and Risk of Incident Heart Failure. J Am Coll Cardiol. 2005:45:2015-21.

29. Tajuddin N, Shaikh A, Hassan A. Prescription omega-3 fatty acid products: considerations for patients with diabetes mellitus. Diabetes Metab Syndr Obes Targets Ther. 2016;9:109-18.

30. Mozaffarian D, Lemaitre RN, King IB, Song X, Spiegelman D, Sacks FM, et al. Circulating Long-Chain $\omega-3$ Fatty Acids and Incidence of Congestive Heart Failure in Older Adults: The Cardiovascular Health Study: A Cohort Study. Ann Intern Med. 2011;155:160.

31. Perini JÂDL, Stevanato FB, Sargi SC, Visentainer JEL, Dalalio MMDO, Matshushita $\mathrm{M}$, et al. Omega-3 and omega-6 polyunsaturated fatty acids: metabolism in mammals and immune response. Rev Nutr. 2010;23: 1075-86

32. Giugliano D, Ceriello A, Esposito K. The effects of diet on inflammation: emphasis on the metabolic syndrome. J Am Coll Cardiol. 2006:48:677-85.

33. Yancy CW, Jessup M, Bozkurt B, Butler J, Casey DE, Drazner MH, et al. 2013 ACCF/AHA guideline for the management of heart failure: a report of the American College of Cardiology Foundation/American Heart Association Task Force on Practice Guidelines. J Am Coll Cardiol. 2013;62:e147-239. 
34. Gastelurrutia P, Lupón J, de Antonio M, Urrutia A, Díez C, Coll R, et al. Statins in heart failure: the paradox between large randomized clinical trials and real life. Mayo Clin Proc. 2012;87:555-60.

35. Kastelein JJP, Maki KC, Susekov A, Ezhov M, Nordestgaard BG, Machielse BN, et al. Omega-3 free fatty acids for the treatment of severe hypertriglyceridemia: the EpanoVa fOr Lowering Very high triglyceridEs (EVOLVE) trial. J Clin Lipidol. 2014;8:94-106.

36. Tousoulis D, Plastiras A, Siasos G, Oikonomou E, Verveniotis A, Kokkou E, et al. Omega-3 PUFAs improved endothelial function and arterial stiffness with a parallel antiinflammatory effect in adults with metabolic syndrome. Atherosclerosis. 2014;232:10-6.

37. von Schacky C. n-3 PUFA in CVD: influence of cytokine polymorphism. Proc Nutr Soc. 2007;66:166-70.

38. Zhao YT, Shao L, Teng LL, Hu B, Luo Y, Yu X, et al. Effects of $n-3$ polyunsaturated fatty acid therapy on plasma inflammatory markers and $\mathrm{N}$-terminal pro-brain natriuretic peptide in elderly patients with chronic heart failure. J Int Med Res. 2009:37:1831-41.

39. Xin W, Wei W, Li X. Effects of fish oil supplementation on inflammatory markers in chronic heart failure: a meta-analysis of randomized controlled trials. BMC Cardiovasc Disord. 2012;12:77.

40. Neff LM, Culiner J, Cunningham-Rundles S, Seidman C, Meehan D, Maturi J, et al. Algal docosahexaenoic acid affects plasma lipoprotein particle size distribution in overweight and obese adults. J Nutr. 2011;141:207-13.

41. Satoh-Asahara N, Shimatsu A, Sasaki Y, Nakaoka H, Himeno A, Tochiya M, et al. Highly Purified Eicosapentaenoic Acid Increases Interleukin-10 Levels of Peripheral Blood Monocytes in Obese Patients With Dyslipidemia. Diabetes Care. 2012;35:2631-9.

42. Gharekhani A, Khatami M-R, Dashti-Khavidaki S, Razeghi E, Abdollahi A Hashemi-Nazari S-S, et al. Effects of Oral Supplementation With Omega-3 Fatty Acids on Nutritional State and Inflammatory Markers in Maintenance Hemodialysis Patients. J Ren Nutr. 2014;24:177-85.

43. da Silva EL. Omega-3 Fatty Acids Improve Lipid Metabolism and Inflammatory Markers in Patients with Heart Failure Undergoing Exercise Training. Ommega Int. 2015;2:1-8.

44. Darghosian L, Free M, Li J, Gebretsadik T, Bian A, Shintani A, et al. Effect of Omega-Three Polyunsaturated Fatty Acids on Inflammation, Oxidative Stress, and Recurrence of Atrial Fibrillation. Am J Cardiol. 2015:115:196-201.

45. Vitelli-Avelar DM, Sathler-Avelar R, Teixeira-Carvalho A, Pinto Dias JC, Gontijo ED, Faria AM, et al. Strategy to assess the overall cytokine profile of circulating leukocytes and its association with distinct clinical forms of human Chagas disease. Scand J Immunol. 2008;68:516-25.

46. Guedes PMM, Gutierrez FRS, Silva GK, Dellalibera-Joviliano R, Rodrigues GJ, Bendhack LM, et al. Deficient regulatory $T$ cell activity and low frequency of IL-17-producing T cells correlate with the extent of cardiomyopathy in human Chagas' disease. PLoS Negl Trop Dis. 2012;6:e1630.

47. Rocha Rodrigues DB, Dos Reis MA, Romano A, de Pereira SA L, de PA TV, Tostes SJ, et al. In situ expression of regulatory cytokines by heart inflammatory cells in Chagas' disease patients with heart failure. Clin Dev Immunol. 2012; 2012:361730.

48. Akl EA, Briel M, You J, Sun X, Johnston BC, Busse JW, et al. Potential impact on estimated treatment effects of information lost to follow-up in randomised controlled trials (LOST-IT): systematic review. BMJ. 2012;344:e2809.

\section{Submit your next manuscript to BioMed Central and we will help you at every step:}

- We accept pre-submission inquiries

- Our selector tool helps you to find the most relevant journal

- We provide round the clock customer support

- Convenient online submission

- Thorough peer review

- Inclusion in PubMed and all major indexing services

- Maximum visibility for your research

Submit your manuscript at www.biomedcentral.com/submit 